\title{
The New Cosmology Arising from the Quantum Pushing Gravity Interaction-The Case of Accelerating Universe
}

\author{
Maurizio Michelini ${ }^{1}$ \\ ${ }^{1}$ ENEA-Casaccia Research Centre, Rome, Italy \\ Correspondence: Maurizio Michelini, ENEA-Casaccia Research Centre, Rome, Italy. E-mail: m_michelini@alice.it
}

Received: July 8, 2013 Accepted: August 1, 2013 Online Published: August 22, 2013

doi:10.5539/apr.v5n5p67 URL: http://dx.doi.org/10.5539/apr.v5n5p67

\begin{abstract}
In 2011 three astronomers were awarded the Nobel Prize for Physics having focussed a correct method of measuring cosmic distances by observation of far Supernovae Ia. Assuming the $\Lambda$ CDM expanding model, they showed that part of the Universe was accelerating, contrary to the original General Relativity predictions. The acceleration was attributed to an Obscure energy linked to the cosmological constant $\Lambda$. This constant was formerly introduced by Einstein in the original G.R. equations to empirically counterbalance the gravitational force to the aim of describing the (known) static universe. After the Hubble's discovery (1929) of galaxy redshifts, which launched the expanding universe, Einstein rejected $\Lambda$. This was the sign that he considered $\Lambda$ a provisional parameter, not the expression of a new physical force. Nevertheless, this parameter is till today taken into account by cosmologists to fit their observations through the $\Lambda$ CDM model. Something is not consequent in the history of the Gravitational theory. The "accelerated expansion" of the universe can be firmly established only through a model independent way. In fact the SNe distances calculated by $\Lambda$ CDM expanding model resulted so large that part of the SNe exploded before the so called Big bang Age, in contrast with the bases of the expansion. The astronomers appear reluctant to claim this splashing result. Something in the Obscure energy is not convincing. Before knowing the astronomer's work, I carried out a research about the physical inadequacy of General Relativity as proper theory of Gravitation, notwithstanding the great success in astronomical observations. The profound reason of this inconsistency is the absence of interaction-waves, contrarily to other fundamental Interactions considered in the Standard Model. From several decades the research attempted to improve this unsatisfactory situation trying to detect the so called gravitational waves coming from cosmic events, but no tangible results have been obtained yet. Actually, the gravitational-inertial waves surely exist, as well as the measured gravitational force. The wavelength I found is of the order of the Planck's length, so adequate tools are necessary for detection. One year ago I published a comprehensive analysis which, recognising the inadequacy of the old kinematic basis of Special relativity, gave rise (Michelini, 2012) to a Relativistic Dynamics re-founded on the new dynamical basis of the Micro-quanta paradigm. The high flux $\phi_{0}$ of micro-quanta filling the space originates, through the mutual-shielding between two particles/masses, the Quantum Pushing gravitational force, whereas at very short distances $\left(<10^{-15}\right)$ the same flux $\phi_{0}$ generates the Strong and Weak nuclear forces. Contrarily to the old Newton's ideas, all these drawing forces do not pull the particles, but push them each other, thus giving rise to the process of Unification of forces. The present paper deals in particular with the Quantum Pushing Gravitation operating on large distances (cosmology), so that between two very distant masses the effect of the mutual scattering on the interposed micro-quanta is not negligible, giving rise to an attenuation factor on the gravitational constant. Quantum Pushing gravitation defines a variable $\mathrm{G}(r)=\mathrm{G} \exp \left(-\Sigma_{o} \mathrm{r}\right)$ showing that parts of Universe at cosmological distances do not feel sensibly the gravitational force. Then the Universe results static, i.e. not expanding. The observed high Hubble's redshifts depend on the interaction of photons that travelled across the micro-quanta giving rise to the redshift $z(x)=\exp \left(x / L_{0}\right)-1$. The SNe measuring method just discovered exponentially increasing redshifs. Trying to explain this fact, the redshfts were introduced in the $\Lambda$ CDM expanding model that produced the large distances resembling an accelerating universe. Several SNe resulted to exist well before the Big bang. Considering this contrast, which inhibits the expanding models, we introduced the observed $\mathrm{SNe}$ redshifts in the Static model, finding that the last SNa $(z=1.91)$ explosion happened 15 Gys ago, a reasonable result. Attributing the Hubble's redshift to the Doppler appears no longer necessary. The static universe, immersed within the micro-quanta, is obviously evolving because the synthesis of heavy nuclei always occurs within the star cores. No arbitrary constant $\Lambda$ has to be introduced in the Static model since the erroneous concept of
\end{abstract}


"gravitational" mass has been previously eliminated through the Quantum Pushing gravity. The main proof of the Static model is the quantitative recognition that the observed Cosmic Microwave Background is due to redshifted photons emitted-with the characteristic black body spectrum-from all stars of the universe.

Keywords: Special and General relativity, Quantum Pushing Gravity, Hubble's law of redshift, Cosmology

\section{Introduction}

The astronomers Perlmutter, Riess and Schmidt (see paragraph 4) showed a correct methodology to measure the distance of far galaxies through the observation of Ia-supernovae with standard luminosity. Here the choice of using the $\Lambda \mathrm{CDM}$ expanding model to explain their observations is not discussed. The $\Lambda$ CDM model considers: a) the luminous matter, b) the Dark matter, c) the empirical parameter $\Lambda$ linked to a cosmic repulsive force due to the Obscure energy. The parameter $\Lambda$ does not pertain to the orthodox Gravitational theory. In any case, whatever the assumed cosmological model, the final prediction of a measured cosmic quantity must result accurate for the expanding as well as the static cosmological model. In particular it is decisive to reproduce the Cosmic Microwave Background (here renamed Cosmic Background Radiation to avoid any confusion with the Micro-quanta), Obviously the correct model is that which gives the right prediction without assuming unexplained hypotheses about the initial universe and without hiding none of the known present phenomena.

The works showing an accelerating universe in contrast with the orthodox G.R. prediction, has been obtained introducing a large term $\Lambda$ on the expanding $\Lambda \mathrm{CDM}$ model. To firmly establish the accelerated expansion of the universe, one has to proof it through a model independent way (Schwartz \& Weinhorst, 2007). G.R. is not put in doubt, because the change regards only the term $\Lambda$, not the original theory. G.R. was successful to supply correct explanations of astronomic observations that imply small effects on the precession of pericentre of planets and binary pulsars, on the photon frequency and the trajectory-bending of photons travelling near large celestial masses (such as the gravitational lenses). These effects justify the four dimensional space metrics adopted in G.R. However, from the viewpoint of Fundamental Physics it is not clear if the original G.R.-i.e. without the empirical parameter $\Lambda$-gives correct cosmological models.

The "discovery" of the accelerating universe in contrast with the G.R. expectations, reset Cosmology to the time when Hubble published his observations about the proportionality between redshift and distance of far galaxies (1929). Before that event, the static universe was considered more realistic than the expanding universe. Let's remark that the first supporter of the static universe was Albert Einstein which, aware that his original equation allowed a spontaneous universal collapse under the gravitational force, introduced an arbitrary cosmological constant $\Lambda$ to balance the gravitation on very large distances. This fact has been neatly recalled by S.M. Carroll: "Einstein was interested in finding static solutions, both due to his hope that General relativity would embody Mach's principle (i.e. matter determines inertia) and simply to account for astronomical data as they were understood at the time" (Carroll, 2011). In the Einstein's thought, the introduction of the cosmological term $\Lambda$ would have changed the Friedmann equations [where $a(t)=R(t) / R_{o}$ is the scale factor of the universe].

$$
H^{2}=(1 / a)^{2}(\mathrm{~d} a / d t)^{2}=(8 \pi / 3) \mathrm{G} \rho+\Lambda c^{2} / 3-\mathrm{k} c^{2} / a^{2} R_{o}{ }^{2}
$$

so as to describe a static universe. In fact the equation admits a static solution (Hubble's constant $H=0$ ) with positive spatial curvature $(\mathrm{k}=1)$ and all parameters $\rho, \Lambda$ non negative. This solution is called the Einstein's static universe (with no redshift).

After the pubblication of the Hubble's cosmological redshift, the situation reversed and the conviction that the universe was really expanding became the most diffuse, because there was no idea of some mechanism producing the photon redshift, excepting the Doppler effect. Einstein rejected hastily the cosmological constant. Prudently, the cosmologists have till now not removed this constant, which is considered necessary to select cosmological models that fit their observations. S. M. Carroll concludes his reflection in this way "However, recent years have provided the best evidence yet that this elusive quantity does play an important dynamical role in the universe" (Carroll, 2011). The tortuous vicissitude of the cosmological constant—proposed and subsequently withdrawn-may be explained if the original Einstein's gravitational equation contained some conceptual bug that was only partially compensated through the empirical parameter $\Lambda$.

To day, I think that the Einstein's sudden rejection of the cosmological term prevented him from recognising (sooner or later) that in G.R. there was a bug inherited from the tenets of the preceding theories. For instance the old Newton's assumption that the gravitational constant $\mathrm{G}$ is uniform across the universe. Up to now no experimental data confirmed it. This old assumption going back to Newton's ideas, inevitably leads to an unstable cosmic equilibrium, which may evolve towards expansion or contraction. When in 1922 the mathematician Friedmann pointed out the instability of the G. R. cosmological equation, Einstein recognised that fact. As a physicist, he 
hypothesised that a possible cosmic collapse (the most disturbing at the time) might be prevented through a repulsive force (temporarily unknown) acting on cosmic scale. The usefulness of the empirical cosmological constant $\Lambda$ resided on the fact that it was considered necessary to guarantee the cosmic equilibrium exhibited by the fixed masses of universe, which gave at the epoch the hope of an answer (through Mach's principle) to the problem of the inertia of bodies. But the cosmic equilibrium so arbitrarily achieved, remained unstable. This appeared clear when Hubble discovered the redshift of galaxies. The expanding universe was welcome and Einstein rejected the constant $\Lambda$, he defined the greatest error in his life. With this franc admission he declared also his inability to individuate the bug hidden in the Newton's gravitation.

Now we know that the misconception of the "gravitational" mass originated a gravitational constant Geverywhere uniform in the universe. On the contrary, the gravitational quantity $\mathrm{G}(r)=\mathrm{G} \exp \left(-\Sigma_{\mathrm{o}} r\right)$ defined by the new Quantum Pushing gravity is exponentially reducing with distance. Quantum Pushing gravity considers only inertial mass and interaction waves (no "gravitational" mass). In conclusion G.R. theory, which gave a precise description of the local astronomical observations, results incorrect on the cosmological scale.

The retard on the recognition of such misconception inside the Newton's law exceeds largely a century since Classical physics ascertained the "gravitational" mass to be quantitatively indistinguishable (up to $10^{-12}$ ) from the inertial mass. That result was correct, so the "gravitational" mass is only a duplicate of the inertial mass. But nobody tried to know the ultimate consequences of that discovery. The reason why the inertial mass of particles, immersed in space filled by micro-quanta, shows drawing properties is the mutual-shielding between particles. At macroscopic level we measure a gravitational force between two masses, but we have no possibility of discovering if the masses of particles are mutually drawing or are pushed each other.

A second bug introduced by the misconception of the "gravitational" mass is the so called unlimited gravitational collapse, which produces a material point having the mass of a star. This is contrary to the Principles of physics. Why the Newton's gravitational force leads to an infinite density and infinite pressure? The reason is evidently linked to the fact that the "gravitational" mass does never loss the property of attracting additional mass (Michelini, 2011). In nature the collapse of massive star happens routinely in the Supernovae which explode expelling great part of the mass and leaving one (or two) neutron stars with mass around the solar mass. Their average density is of the order of $10^{18}$ and correspondingly the radius is small $\mathrm{R} \approx 10^{4}$. Defining this phenomenon an unlimited gravitational collapse is wrong. Nature avoids the unlimited gravitational collapse, but all theories that define the Gravit. mass undergo it. In the Quantum pushing gravity the unlimited collapse is theoretically prevented because the maximum gravitational force of a mass $\mathrm{M}$ takes place on the peripheral nucleons (cross section $\sigma$ ) upon which is applied, on a very little fraction $y=\Delta \sigma / \sigma$ of cross section, the high radiation pressure $p_{\mathrm{o}}=E_{\mathrm{o}} \phi_{\mathrm{o}} / c \approx 2.4 \times 10^{61}$ (see Equation 2.2) that equals the energy density of micro-quanta. Since the gravitational force on the nucleon is $F$ $=\mathrm{GM} m / \mathrm{R}^{2}$, the little fraction $\Delta \sigma$ is defined by $F=\Delta \sigma p_{0}$. In other words the mutual-shielding operated on the peripheral nucleon by the $\mathrm{N}=M / m$ nucleons constituting the mass $\mathrm{M}$, originates on the cross section a little area $\Delta \sigma$ upon which do not collide the micro-quanta escaping from the mass. This produces on the external side of the peripheral nucleon the Gravitational force $F=\Delta \sigma p_{0}$ due to the micro-quanta radiation pressure. Let's now express the fraction $y=\mathrm{GM} m / \mathrm{R}^{2} \sigma p_{\mathrm{o}}$ which, substituting the ratio $\sigma / m=\mathrm{A}_{\mathrm{o}}$ (see Equation 2.2) gives the fraction $y$ $=\mathrm{GM} / \mathrm{R}^{2} \mathrm{~A}_{\mathrm{o}} p_{\mathrm{o}}$. This fraction takes high values $\left(y \approx 2 \times 10^{-39}\right)$ for neutron stars whose ratio $\mathrm{M} / \mathrm{R}^{2}$ reaches values up to $2 \times 10^{22}$, while the obscure Supermasses at the centre of galaxies take even greater values of the ratio. The theoretical maximum attainable value of the fraction is $y=1$. In this limiting case the collapse is also arrested since the gravitational internal pressure cannot ever attain the radiation pressure $p_{0}$. No infinite gravitational pressure, no unlimited collapse.

Coming back to the early cosmology following the Hubble's observations, the scientific community split into opposite opinions. The expanding universe stood obviously on the Doppler effect. The static universe model considered the redshift was due to a continuous loss of photon energy through interaction with a medium filling the space. For long time this line of thought didn't find a physical explanation since the postulate of empty space was dominant. In recent times the conviction that the physical interactions transmit through waves at light velocity became predominant, so it was remarked that G.R. gravitation is the only theory sine interaction waves, contrary to other fundamental forces described in the Standard Model of particles. Only recently the "gravitational" mass has been fully recognised not to be the source of the gravitational field thanks to the Micro-quanta paradigm, where the space is filled by micro-quanta that transmit the interactions. This paradigm has been firstly verified by discussing some unexplained phenomena (Michelini, 2010) that in the past have been dropped since they did not fit the classical paradigms. Besides the Gravitation and the inertial forces, the Micro-quanta paradigm explains also the origin of other fundamental forces, such as the Strong and Weak nuclear drawing forces (Michelini, 2012). 


\section{The Equation of Pushing Gravity at Cosmological Distances}

Pushing gravitation arises from the mutual shielding between two particles immersed in the flux $\phi_{0}$ of micro-quanta with energy $E_{0}$. In that following we resume briefly the physical concepts that lead to the definition of the Quantum pushing gravity equation (Michelini, 2012). Due to mutual shielding, a beam of quanta disappears along a tiny tunnel (section $\Delta \sigma$, length $r$ ) placed on the line joining the particles (missing beam)

$$
\psi(r)=\Delta \sigma \phi_{0}(\Delta \sigma / 2 \pi r)(\text { quanta } / \mathrm{sec})
$$

where $\Delta \sigma=\left(\mathrm{K}_{\mathrm{o}}\right)^{1 / 2} \sigma$ is the fraction of cross section upon the two particles which is not reached by quanta. Substituting $\Delta \sigma$ and multiplying the beam $\psi(r)$ by the momentum $\left(2 \mathrm{E}_{o} / \mathrm{c}\right)$ that a recoiling micro-quantum gives up to each particle, one gets the first order gravitational force which pushes each other the particles

$$
\boldsymbol{F}_{1}(r)=\left(2 \mathrm{E}_{\mathrm{o}} / \mathrm{c}\right) \psi(r)=2 \mathrm{~K}_{\mathrm{o}} \sigma p_{\mathrm{o}}\left(\sigma / 2 \pi r^{2}\right)
$$

where $\mathrm{K}_{\mathrm{o}} \approx 3.93 \times 10^{-51}$ is the inverse of the number of simultaneous collisions on a nucleon and $p_{0}=\mathrm{E}_{\mathrm{o}} \phi_{\mathrm{o}} / \mathrm{c} \approx$ $2.4 \times 10^{61}$ is the Energy density of the micro-quanta flux. All quantities are given in SI system.

Substituting in Equation (2.2) the expression of the gravitational constant

$$
G=p_{0} K_{0} A_{0}^{2} / \pi=6.6710^{-11}
$$

where $\mathrm{A}_{\mathrm{o}}=\sigma_{\mathrm{i}} / \mathrm{m}_{\mathrm{i}} \approx 4.7 \times 10^{-11}$ is constant for all massive particles (Michelini, 2012), we obtain the newtonian gravitation between two particles, typically nucleons (cross-section $\sigma \approx 7.85 \times 10^{-38}$ ) that represent $99.97 \%$ of the atomic mass

$$
\boldsymbol{F}_{l}(r)=G m^{2} / r^{2} .
$$

Let's us recall that the constant $A_{o}$ with dimensions $\left[\mathrm{m}^{2} / \mathrm{kg}\right.$ ] was searched in the ' 30 by W. Heisenberg, the physicist that enounced the Uncertainty principle linked - at the epoch - to the physical impossibility of seeing microscopic objects whose size was less than the wavelength $\lambda=\mathrm{h} / \Delta \mathrm{p}$ of the scattered photons. More recently the application of high-momentum electrons in the electronic microscopes reduced strongly the size of the observable objects. The introduction of the Micro-quanta might require a possible redefinition of the principle due to the very little wavelength of these quanta which operate with the modality of $\mathrm{N}_{\mathrm{i}}=\sigma_{\mathrm{i}} \phi_{0} \tau_{\mathrm{o}}$ simultaneous collisions upon a particle. The use of the classical gravitational notation does not obviously reject the modified metrics of G.R. which permits us to explain some small effects in astronomical observations linked to the finite velocity of the light signals or to the deviation of their trajectory near a large mass (for instance the gravitational lenses). The aim to choice the classical notation depends only on the fact that, operating at cosmological distances, we may adopt the euclidean space because the small local effects of light rays become negligible.

It has been suggested (Michelini, 2012) that the correct Pushing gravitation shows an exponential reduction along very large distances due to the presence of mutual scattering (whose macroscopic self-scattering cross-section is $\Sigma_{\mathrm{o}}=\sigma_{\mathrm{o}} \phi_{\mathrm{o}} / \mathrm{c}$, where $\sigma_{\mathrm{o}}$ is the quantum cross section) between the micro-quanta interposed between two masses. As we shall see, a very large distance means comparable with the mean free path $L_{o}=1 / \Sigma_{0}$ of micro-quanta. Let's describe a quantum colliding with another quantum (cross-section $\sigma_{0}$ ) which is scattered within the solid angle $\gamma(r)$ $=\left(\sigma / 2 \pi r^{2}\right)$ towards the other particle at distance $r$. The micro-quanta scattered from the small volume element of the tiny tunnel delimiting the missing beam $\left(\Delta \sigma \Sigma_{0} \phi_{0} \mathrm{ds}\right)$ multiplied by the attenuation $\exp \left(-\Sigma_{0} s\right)$ due to the interposed quanta, may be obtained integrating along the distance $r$. Recalling that each recoiling quantum gives up the particle the momentum $2 \mathrm{E}_{\mathrm{o}} / \mathrm{c}$, the Pushing gravity force between two nucleons at a distance $r$ becomes

$$
\boldsymbol{F}(r)=\left(2 E_{\mathrm{o}} / \mathrm{c}\right) \mathrm{K}_{\mathrm{o}} \sigma \gamma(r)\left[\phi_{\mathrm{o}}-\int_{0}^{r} \Sigma_{\mathrm{o}} \phi_{\mathrm{o}} \exp \left(-\Sigma_{\mathrm{o}} s\right) \mathrm{d} s\right]
$$

Integrating and substituting $\gamma(r), \mathrm{A}_{\mathrm{o}}=\sigma_{i} / m_{i}$ and $p_{\mathrm{o}}=E_{\mathrm{o}} \phi_{\mathrm{o}} / \mathrm{c}$, one gets the correct Pushing gravity force between two nucleons

$$
\boldsymbol{F}(r)=\mathrm{K}_{\mathrm{o}} \mathrm{A}_{\mathrm{o}}^{2} p_{\mathrm{o}}\left(m_{\mathrm{o}}{ }^{2} / \pi r^{2}\right) \exp \left(-\Sigma_{\mathrm{o}} r\right)
$$

Substituting the newtonian gravitational constant (Equation 2.3) gives-with usual notations-the Quantum Pushing force at cosmological scale between two masses made of different number of nucleons

$$
\boldsymbol{F}(r)=\mathrm{G} \exp \left(-\Sigma_{\mathrm{o}} r\right) M_{1} M_{2} / r^{2} .
$$

This equation defines a variable gravitational constant between two masses

$$
\mathrm{G}(r)=\mathrm{G} \exp \left(-\Sigma_{\mathrm{o}} r\right)=\mathrm{G} \exp \left(-r / L_{o}\right)
$$


where $\mathrm{L}_{0}$ is the mean free path across the flux of micro-quanta. The variation of $\mathrm{G}(\mathrm{r})$ is negligible within the solar system and has possibly some significance within our Galaxy. Only on the cosmological scale the value of G(r) tends to zero, so two very distant galaxies are not gravitationally bound. Newton's gravity and the cosmological models assumed by G.R. consider that all matter in the universe is source of gravitational field, so they become inaccurate when $r$ is comparable to $L_{0}$. Viceversa, when $r>L_{o}$ the very low gravitational force on the Universe-at-large makes quasi-static the dynamical equations of motion for the whole universe. Let's recall that the inertial forces (i.e. the dynamical equations) originate from the motion of masses across the M-Q flux, so they exist only within the Cloud of micro-quanta that envelopes the universe of matter. In this conceptual frame we may affirm that the material universe becomes "static", although each component (galaxies, stars, planets, etc) follows its spatial evolution. Obviously, the static universe implies that Hubble's redshift does not depend on Doppler.

\section{Determination of the Mean Free Path of Photons}

Knowing the macroscopic cross-section $\Sigma_{0}=\sigma_{0} \phi_{0} / \mathrm{c}$ of mutual scattering among micro-quanta permits us to calculate the micro-quantum cross-section $\sigma_{0}$. This quantity is very small. We know a procedure to determine the cross section of ordinary electromagnetic photons based on the definition of their equivalent mass respect to the inertial properties of wave packages with energy $h v$

$$
m_{\mathrm{ph}}=h v / c^{2}
$$

This mass is recognised to produce the gravitational effects observed on the photons travelling near a star mass. Recalling the constant $\mathrm{A}_{o}$ we may infer that the cross section of photons is

$$
\sigma_{\mathrm{ph}}=\mathrm{A}_{\mathrm{o}} m_{\mathrm{ph}}=\mathrm{A}_{\mathrm{o}} h v / c^{2} \text {. }
$$

The photon mean free path $L_{\mathrm{ph}}=m / \sigma_{\mathrm{ph}} \delta_{\mathrm{u}}$ across the average density of matter in the universe $\left(\delta_{\mathrm{u}} \approx 10^{-27}\right)$ may be calculated for the diffused "visible" photons coming from stars $\left(h v \approx 4 \times 10^{-19}\right)$ which show a cross section $\sigma_{\text {ph }} \approx$ $2.1 \times 10^{-46}$. The resulting $L_{\mathrm{f}} \approx 6 \times 10^{45}$ is many orders of magnitude higher than the so called visible radius $\mathrm{R}_{\mathrm{v}}$ of the universe, which is the distance of a far galaxy whose redshift becomes so high that photons can be detected with difficulty. Adopting the Hubble's constant derived from the observations of the WMAP satellite, the explored radius extends up to $\mathrm{R}_{\mathrm{v}} \approx 1.7 \times 10^{26}$. Only recently it was pointed out (Lineweaver et al., 2011) that in expanding universe the farthest galaxy attains (in co-moving coordinates) the velocity of light after 46 billions years from Big bang, that is 3.4 times the believed age of universe. At intermediate time-distances, light reaches the receding galaxies, but the photon energy is reduced by Doppler redshift. Let's now calculate the visible radius in the static universe. Obviously we have to consider explicitly the loss of photon energy by redshif $h v=h v_{0} /(\mathrm{z}+1)$ which, by virtue of Equation (4.3) becomes $h v(x)=h v_{0} \cdot \exp \left(-x \Sigma_{0}\right)$. In the static universe the average photon travel weighted on the redshifted photon energy gives the mean free path

$$
L_{\mathrm{o}}=\int_{0}^{X} x h v_{\mathrm{o}} \exp \left(-x \Sigma_{\mathrm{o}}\right) \mathrm{d} x / \int_{0}^{X} h v_{\mathrm{o}} \exp \left(-x \Sigma_{\mathrm{o}}\right) \mathrm{d} x=1 / \Sigma_{\mathrm{o}}\left[1-\exp \left(-\mathrm{X} \Sigma_{\mathrm{o}}\right)\right]
$$

which reduces to $L_{\mathrm{o}}=1 / \Sigma_{\mathrm{o}}$ when $X>L_{\mathrm{o}}$. X is the largest distance from which we may detect some signals. For instance the signals received from the farthest stars (average emission temperature $\mathrm{T}_{s} \approx 5700 \mathrm{~K}$ ) share the Cosmic Background Radiation $\left(\mathrm{T}_{C B R}=2.73 \mathrm{~K}\right.$ ). This cosmic radiation shows an average redshift (see paragraph 6) around $z$ $=\left(T_{S} / T_{C B R}\right) \approx 2087$. This means that the extension $X$ of the static universe does not depend on the interaction photons/matter, but is essentially due to degradation of the photon energy by collisions with micro-quanta. This explanation don't exist in the expanding universe because photons travel within an empty space. In other words, considering a reduction in the photon energy by a factor $z_{0}=2087$, we may enlarge the explored universe by capturing larger fluxes of degraded photons, as Penzias and Wilson made in 1964. From Equation (4.4) we obtain $X=L_{0} \ln \left(1+\mathrm{z}_{\mathrm{o}}\right) \approx 7.64 L_{\mathrm{o}}$, so the distance $X$ is about 7.64 times the present visible radius. This means we receive signals coming from $\Delta \mathrm{t}=7.64 L_{\mathrm{o}} / c=108$ billion light-years (see paragraph 6). What about the Olbers' paradox? The receding luminous galaxies of the expanding universe explain only part of the Olbers' paradox. The full explanation of the nocturnal darkness comes from the proof (see paragraph 6) that the whole radiation emitted by all stars of the universe is redshifted by the interaction between photons and flux of micro-quanta, giving rise to the CBR radiation. Olbers was right: there is something in space that prevent the nocturnal sky be shining.

\section{The Interaction between Photons and Flux of Micro-Quanta (Redshift)}

Quite different is the collision between two micro-quanta, since they are single elementary quanta, not complex objects as photons. An instructive way to face the problem may be the calculation of the mutual scattering between the photons of Cosmic Background Radiation (equivalent temperature $\mathrm{T}_{\mathrm{o}}=2.73^{\circ} \mathrm{K}$ ) which shows a flux $\phi_{\mathrm{CBR}}=8.1 \times 10^{16}$ and an average energy $\mathrm{kT}_{\mathrm{o}}$ equal to $3.77 \times 10^{-23}$. Calculating the cross section $\sigma_{\mathrm{CBR}} \approx 2 \times 10^{-50}$, the 
m.f.p. among the CBR photons results $L_{\mathrm{CBR}}=\mathrm{c} / \sigma_{\mathrm{CBR}} \phi_{\mathrm{CBR}} \approx 1.8 \times 10^{41}$, that is lower than the m.f.p. of visible photons across the average density of matter. In the case of micro-quanta the m.f.p. $L_{0}=\mathrm{c} / \sigma_{0} \phi_{0}$ depends on the flux $\phi_{0}>>$

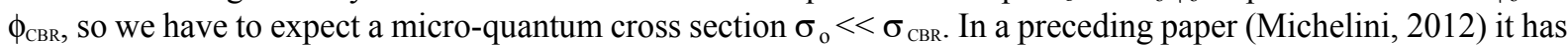
been proposed that photons are packages of Micro-quanta which are "modulated" by excited electrons jumping between two atomic energy levels. This picture makes clear why all photons generated by accelerated electric charges move in space with the same velocity of the micro-quanta beams. This explanation is not trivial because we don't believe that the empty space assumed by G.R. (characterised locally by the curvature) contains physical tools which determine everywhere the same velocity of all photons.

Assuming that the photon energy is due by a great numbers $\mathrm{N}_{\mathrm{o}}=\mathrm{h} v_{\mathrm{o}} / \Delta \mathrm{E}$ of polarised micro-quanta linked each other by little energy $\Delta \mathrm{E}<<\mathrm{E}_{0}$, we have to explain the problem of photon propagation across the flux of Micro-Quanta. It is conceivable that each constituent quantum (cross section $\sigma_{\mathrm{o}}$ ) undergoes a collision with the flux $\phi_{0}$ of micro-quanta which scatters it from the photon assembly, so the number of constituent quanta $\mathrm{N}(x)$ reduces along the travel. Putting $\Sigma_{0}=\sigma_{0} \phi_{0} / c$ the macroscopic cross section of scattered micro-quanta, one writes

This equation shows that

$$
\mathrm{dN}(x) / \mathrm{d} x=-\Sigma_{0} \mathrm{~N}(x) .
$$

$$
\mathrm{N}(x)=\mathrm{N}_{\mathrm{o}} \exp \left(-\Sigma_{\mathrm{o}} x\right) .
$$

Substituting in this equation $\mathrm{N}(x) / \mathrm{N}_{\mathrm{o}}=\mathrm{h} v(x) / \mathrm{h} v_{\mathrm{o}}$ one obtains $\exp \left(-\Sigma_{0} x\right)=v(x) / v_{0}$. Recalling the definition of redshift $z=\left[v_{0} / v(x)\right]-1$ we have

$$
z(x)=\exp \left(\Sigma_{0} x\right)-1=\exp \left(x / L_{o}\right)-1 .
$$

Let's notice that for distances $x$ much smaller than the mean free path $L_{o}$ this equation gives

$$
z \approx x / L_{o}
$$

which is the original Hubble's law before the expanding universe interpretation $z \approx x H_{o} / c$ prevailed. Equation (4.3) expresses the redshift of photons travelling within the Static universe filled by micro-quanta. Static universe means obviously non-expanding. This universe shows all characteristics of the real evolving universe. Up to now we supposed that the scattering cross section $\Sigma_{0}$ linked to the flux is uniform within the material universe. However we know that its radius $R_{\mathrm{m}}$ is finite and has been estimated $R_{\mathrm{m}} \approx X$ from the circumstance (paragraph 3 ) that a fraction of the $\mathrm{CBR}$ radiation comes from the farthest stars of the universe. If the material universe is finite, even the Grand Cloud of micro-quanta has a finite radius. This means that the micro-quanta flux is declining at distances around $R_{\mathrm{m}}$ and the scattering cross section $\Sigma(x)=\sigma_{\mathrm{o}} \phi(x) / \mathrm{c}$ depends on the flux $\phi(x)$.

This outline takes full significance in what follows, where it is found the value of the mean free path $L_{o} \approx$ $1.35 \times 10^{26}$, giving a clear idea of the radius $R_{\mathrm{m}} \approx L_{\mathrm{o}} \ln \left(1+\mathrm{z}_{\mathrm{o}}\right)=7.64 L_{\mathrm{o}}$ of the Static universe. However we don't know if $\phi_{\mathrm{o}}(x)$ shows a tangible declining within the distance $R_{\mathrm{m}}$. The condition that the material universe be contained within the Grand Cloud of micro-quanta with radius $R_{\mathrm{u}} \gg>R_{\mathrm{m}}$ is necessary to guarantee the presence of the fundamental forces (gravitation, inertial forces, etc) even on the farthest galaxy. In paragraph 9 we try to face the problem of the size of the Grand Cloud of micro-quanta.

Recalling that $\Sigma(x)=\sigma_{0} \phi(x) / \mathrm{c}$ let's now define in general the optical distance

$$
\xi(x)=\int_{0}^{x} \Sigma_{o}(x) \mathrm{d} x=\int_{0}^{x} \sigma_{0} \phi(x) \mathrm{d} x / \mathrm{c}=x \underline{\Sigma}(x)
$$

where $\underline{\Sigma}(x)$ is the average cross section along the distance $x$. As an obvious consequence the fundamental Equation (4.3) may be formulated in general introducing the optica

$$
\xi(x)=x \underline{\Sigma}(x)=\ln [1+z(x)] .
$$

The optical distance across the flux of micro-quanta $\phi(x)$ is fundamental in the Static universe model to calculate the distances. In particular it will be shown in par.9 that the average cross section does not change sensibly within the material universe, that is $\underline{\Sigma}(x) \cong \Sigma_{0}$. This enables to express in Static model the distance in terms of redshift, that is a quantity that can be measured

$$
x_{\mathrm{st}} \cong L_{\mathrm{o}} \xi(x)=L_{o} \ln [1+\mathrm{z}(x)]
$$

where $L_{0} \cong 1 / \Sigma_{0}$ is the micro-quanta m.f.p. 


\subsection{Fundamentals of the Static Model of Universe}

We recall briefly the fcharacteristics of the Static model of universe.

1) The Cosmological Equations do not contain the parameter $\Lambda$, whereas the gravitational constant is substituted by $\mathrm{G}(r)=\mathrm{G} \exp \left(-\Sigma_{0} r\right)$ defined by the Quantum Pushing gravitational force.

2) The cosmological photon redshift $z(x)$ arises from the interaction between single photons travelling across the micro-quanta flux along the distance $x$ (Equation 4.3). Of course the Doppler redshifts and gravitational redshifts are measured apart for sources with proper gravitational motion.

3) The distance of a celestial body with measured cosmological redshift (Equation 4.4) is given by $x_{s t}=L_{o} \ln (1+\mathrm{z})$, where $L_{\mathrm{o}}$ is the m.f.p. of the micro-quanta flux, a quantity presently known.

4) The proof of the Static model resides in the demonstration (see paragraph 6) that collecting all star radiation reaching the Earth [radiation beams with the black body spectrum undergo the redshift $(1+z)^{3}=\exp \left(3 \Sigma_{0} x\right) \operatorname{coming}$ from stars at distance $x$ ] we find a degraded power flux which may likely show the same value (Equation 6.8) found by Penzias \& Wilson in 1964 when they discovered the Cosmic Microwave Background radiation.

5) The conceptual reliability of the Static distances $x_{\mathrm{st}}$ comes from the dependence on measured quantities only: $L_{\mathrm{o}}$ and redshift $z$. The problem of determining the m.f.p. $L_{0}$ can be solved through the known cosmological observations. It is well known that when $z<<1$ (near Universe) the distance in Static model equals the distance in Expanding model $x_{\mathrm{st}} \cong x$. For any given source, the redshift $z \cong\left(H_{\mathrm{o}} / \mathrm{c}\right) x$ in expanding universe equals the redshift $z \cong x_{\mathrm{st}} / L_{\mathrm{o}}$ in static universe (Equation $4.3 \mathrm{a}$ ). The result is

$$
z / x \cong 1 / L_{\mathrm{o}}=\left(H_{\mathrm{o}} / \mathrm{c}\right) \text {. }
$$

Let's assume the Hubble's constant obtained by satellite measurements [Planck: $H_{\mathrm{o}}=67.9$; WMAP: $H_{\mathrm{o}}=70.0(\mathrm{~km}$ $\left.\mathrm{s}^{-1} \mathrm{Mpc}^{-1}\right)$. The intermediate value gives

$$
\left(H_{\mathrm{o}} / c\right) \approx 0.740 \times 10^{-26}
$$

which recalling Equation (4.5) gives the m.f.p.

$$
L_{\mathrm{o}} \cong\left(\mathrm{c} / H_{\mathrm{o}}\right) \approx 1.35 \times 10^{26} .
$$

We consider this result reliable for a more fundamental reason. From the analysis of the All Star Redshifted radiation $\mathrm{p}_{\text {ASR }}$ performed in par. 6 it appears that this value of $L_{o}$ agrees (Equation 6.8) with the measured CBR power flux. This represents a tangible proof of the physical nature of the micro-quanta mean free path $L_{0}=1 / \Sigma_{0}$ within the universe filled by stars from which comes the CBR power flux.

\subsection{The Case of Accelerating Universe}

The recent observations of the luminosity of distant Supernovae Ia (often described as the "discovery" of an Accelerating Universe) make interesting to compare the Static model distances with the distances yielded by the $\Lambda \mathrm{CDM}$ expanding model adopted by the astronomers. The choice of an expanding model was expected in the era of the Big bang model. Less expected was the choice of adding to the original G.R. equations an arbitrary value of the cosmological constant $\Lambda$.

A series of measurements of SNe distances was due to the Supernova Cosmology Project (Perlmutter et al., 1999), while an other series of redshift measurements and $\Lambda$ CDM-calculated distances (Hubble's diagram) is reported on Figure 4 (Riess et al., 2004) regarding two different sets of supernovae Ia observations obtained from ground-based telescopes and from the Hubble Space Telescope (HST). The two sets agree and individuate a fitting curve with redshift ranging from $0<\mathrm{z}<2$, where the HST measurements prevail at high redshifts. Some values of the fitting curve are reported in the following Table 1 to make clear our considerations. The last two rows are obtained directly from the Figure 22 (Schmidt, Riess et al., 2005) which use the same data of Figure 4 in a Hubble's diagram with $\log (\mathrm{z})$ on the abscissa, thus giving easily readable redshifts at low values.

In the same Table are reported, to the aim of doing synoptic comparison, the Static distances defined through Equation (4.4) $x_{\mathrm{st}}=L_{\mathrm{o}} \ln (1+z)$. The time of photon travel along the $\Lambda \mathrm{CDM}$-calculated distances $x$ is calculated according to Special relativity. 
Table 1. Redshifts and distances taken from Figure 4 showing the fitting curve of two series of measurements obtained from observations of SNe Ia made on ground telescopes and by Hubble Space Telescope (A. Riess et al., 2004). To make a comparison we reported the Static distances calculated for the same $\mathrm{SNe}$ in the last column

\begin{tabular}{ccccc}
\hline SNe Redshift & Present distance $x$ & S.R. photon travel & Optical distance & Static Distance $x_{\text {st }}$ \\
\hline$z$ & ACDM model & $\Delta t=x / c$ & $\xi(x)=\ln (1+z)$ & Static model \\
\hline$\left(^{*}\right) 1.91$ & $\approx 4.4 \times 10^{26} \mathrm{~m}$ & $\approx 46 \mathrm{Gys}$ & 1.067 & $1.44 \times 10^{26} \mathrm{~m}$ \\
1.76 & 3.88 & 40.4 & 1.016 & 1.37 \\
0.83 & 1.55 & 16.1 & 0.604 & 0.816 \\
0.20 & 0.306 & 3.19 & 0.182 & 0.246 \\
0.10 & 0.159 & 1.65 & 0.0953 & 0.128 \\
0.07 & 0.101 & 1.05 & 0.0676 & 0.0913 \\
0.02 & 0.0268 & 0.28 & 0.0198 & 0.0267 \\
0.01 & 0.0134 & 0.14 & 0.00995 & 0.0134 \\
\hline
\end{tabular}

Note $(*)$ - This measurement is not reported in Figure 4 (cit.). The Supernova with $\mathrm{z}=1.914$ has been recently observed by A. Riess, D. Jones, S. Rodney. HUBBLE SPACE TELESCOPE-Press release: April 4, 2013 (NASA/ESA, HST, 2013). Here is reported the $\Lambda$ CDM calculated distance, corresponding to the redshift $\mathrm{z}$ $=1.914$ on the fitting curve (Figure 4 and Figure 22).

The Table presents the distances $x$ calculated by the $\Lambda$ CDM expanding model, showing that the removal of Supernovae increases notably with redshift. This generates the so called accelerating universe. These facts show some important physical consequences.

A) The distances $x$ (i.e. between the present positions of Supernovae) calculated assuming the expanding $\Lambda \mathrm{CDM}$ model, show the increasing removal of galaxies. Cosmological models calculates the time spent by photons to reach the observer at velocity of light. Someone writes of galaxies receding with velocity greater than $c$ putting forwards the argument they are involved in the "expansion of space". This opinion ignores that the gravitational force, the pressure of Obscure energy and the SNe luminous flash, all travel at the velocity $c$ of micro-quanta. It ignores also that the Inertial forces on the accelerating galaxies do not come from old formulae linked to the frame of coordinates, but come from the physical interaction of accelerating masses with the flux of micro-quanta. The G.R. Cosmology is ruled out by the Quantum Pushing Gravity. Conversely, Special relativity is reinforced by the Micro-quanta Paradigm (Michelini, 2012). S.R. was never contradicted about the velocity of photons and particles. It may be useful to recall the story of neutrinos speeder than light.

B) The photon travel times (calculated according to Special Relativity) shown in Tablel exceed largely (for redshift higher than $\approx 0.8$ ) the 13.7 Gys of the Big bang age, which could be ruled out if the calculated distances are correct. But in the authors' papers there is no mention of this splashing result. The contrast between the Big bang model and the $\Lambda \mathrm{CDM}$ model entitles to calculate within the Static model the $\mathrm{SNe}$ observed data obtaining the distances $x_{\mathrm{st}}$ reported on the last column. These distances, obtained through the redshift of photons interacting with the micro-quanta, present a remarkable reliability because are given only through known quantities ( $L_{\mathrm{o}}$ and redshift). Finally they do not contrast with the current knowledge of the universe.

C) At very small redshifts $\mathrm{z}<<0.01$ the distances from the $\Lambda \mathrm{CDM}$ model and from Static model are equal, that is $x_{\mathrm{st}}=x$. As shown by Equation (4.7) one obtains the value of $L_{\mathrm{o}} \cong\left(c / H_{\mathrm{o}}\right) \approx 1.35 \times 10^{26}$ which is engraved in the Hubble's radius measured by WMAP and Planck satellites.

The epoch of the SNe explosion is generally not published by the astronomers. In the Press release: 4 April, 2013 about the last discovered Supernova Ia, the Hubble Space Telescope informs that this supernova exploded more than 10 Gys ago (NASA/ESA, HST, 2013). This indication is in contrast with the $\approx 46$ Gys of the photon travel $\Delta t$ $=x / c$ along the distance $x$ shown in the Hubble's diagram (Table 1) built by the astronomers using the $\Lambda$ CDM model. This is a proof that the Expanding model is in crisis. 
Referring to the same supernova $z=1.91$, Table 1 shows the Static model distance $x_{\mathrm{st}}=L_{o} \ln (2.91)=1.44 \times 10^{26}$ which is equivalent to a photon travel of $\approx 15$ Gys ago. The Static model is much larger than $1.44 \times 10^{26}$ metres, but it does not require that Universe always existed. Obviously in Static universe the SNe ages have no relation with the Big bang age. It is evident the great importance of the initial distances (i.e. at the time of SNe explosion) of the observed Supernovae. It's a pity that the astronomers didn't publish the initial distances. Comparison with the Static distances might result interesting.

The reliability of the Static distances $x_{\mathrm{st}}$ comes from their dependence on measured quantities only. As anticipated in the "Fundamentals of the Static model", the universal value of the micro-quanta mean free path has been found equal to $L_{\mathrm{o}}=\left(\mathrm{c} / H_{\mathrm{o}}\right) \approx 1.35 \times 10^{26}$ using the most recent cosmological results (Planck and WMAP satellites). We consider this value reliable for a more fundamental reason. From the analysis of the All Star Redshifted radiation $\mathrm{p}_{\mathrm{ASR}}$ performed in par. 6 it appears that this value agrees with the measured CBR power flux, thus obtaining a independent proof.

\subsection{The Misleading Expanding Models}

The structure of the Static model is hardy respect to the structure of the $\Lambda \mathrm{CDM}$ model, where the distance depends on the poorly known parameter $\Lambda$. We cannot end our considerations on the role of the cosmological model adopted by the astronomers without observing that in the era of the Big bang model it is expected to test against an Expanding model. Less expected is the choice of defining a parameter $\Lambda$ linked to an Obscure energy whose nature does not appear yet defined. Why to restrict the field? Even to day there are researchers which do not exclude a Static model, so it may be useful to test the SNe measuring method also against a definite Static model.

It appears interesting to verify how the Expanding model distance $(x)$ is linked to the Static model distance $\left(x_{\mathrm{st}}\right)$. To this aim we repeat (using the same experimental optical data) the operation of calculating the SN distances in the Static model considering the redshift suffered by the SNe photon beam travelling across the micro-quanta up to the observer. This redshift takes the value $(1+z)^{3}$ (see Equation 6.3a) for radiation with black body spectrum, such as the stellar radiation. Then the ratio between the Supernova intrinsic luminosity $\mathrm{L}_{\mathrm{SN}}$ and the strength of the signal $f$ $\left(\mathrm{w} / \mathrm{m}^{2}\right)$ observed at a distance $x_{s t}$, results

$$
\left(\mathrm{L}_{\mathrm{SN}} / 4 \pi f\right)=x_{\mathrm{st}}^{2}(1+\mathrm{z})^{3} \text {. }
$$

This experimental ratio is the same obtained in the Expanding model that considers the redshift $(1+z)$ given by Doppler (apart the influence that may be due to the parameter $\Lambda$ )

$$
\left(\mathrm{L}_{\mathrm{sN}} / 4 \pi f\right)=x^{2}(1+z)
$$

where $x$ is the expanding distance. Equating these two expressions and taking the squared roots, one has the relation between the SNe distances calculated both in the Expanding and in the Static model

$$
x=x_{\text {st }}(1+z) .
$$

This result shows with clarity the great difference that originates from calculating the $\mathrm{SNe}$ distances adopting two different models. Which is the most reliable distance of SNe? The Expanding model leads to the destructive result that the calculated distances contrast with the Big bang model, which gave a definite basis to the cosmological Expanding model, at least within the orthodox Cosmology (i.e. without the term $\Lambda$ ).

To a first analysis Equation (4.2c) links two model-dependent distances. So it entitles to make the comparison and the physical evaluation of SNe expanding distances $x$. The Static model stands on the fact that the correct Gravitational theory requires the presence of specific interaction waves (micro-quanta), particularly in the case of Cosmology, where it changes the uniform gravitational constant G. The expression of the static distances $x_{\mathrm{st}}=$ $L_{0} \ln (1+z)$ was obtained considering the redshift due to the photon interaction with the flux of micro-quanta. This phenomenon was missing in all cosmological models due to the postulate of empty space adopted in G.R. a Recently a comprehensive study about different ways of calculating the Hubble's constant in a euclidean universe (Zaninetti, 2010) showed that two mechanisms (different from Doppler) may be invoked in a Static universe to explain the galaxy redshift. The first mechanism is the Plasma redshift. As an example we recall the Cooling phase of the Big bang, where the photons emitted by high-temperature particles were redshifted up to millimetric cosmic waves (CMB). Which was the agent of this redshift? Because Doppler is excluded, one has to search for some phenomenon different from the classical "cooling" which requires the radiation be in equilibrium with a cooling material cavity. The second mechanism comes from the research about the photon interaction with the diffuse gases in the intergalactic space (Dispersive Extinction theory). This theory shows that the absorbed energy is function of the distance travelled by photons within the medium (Wang, 2011), thus introducing an interpretation 
of the cosmic redshift different than Doppler. Obviously these mechanisms hold in some regions of the universe. Conversely, the flux of micro-quanta explains the universal character shown by the cosmological redshift.

Assuming the Static distances are correct, we may search about their relation with the distances of SNe computed through the $\Lambda \mathrm{CDM}$ model. As we previously recalled, the initial distance of any SNa is conceptually defined with precision, so we renew the invitation to the astronomers to publish their SN initial distance.

To give a definite basis to a quantitative comparison, we computed the SNe artificially expanded distances $x^{\prime}=x_{\mathrm{st}}$ $(1+z)$ given by Equation (4.2c) which stands on the basis of the conservation of energy of the photon beams expressed in the previous equations. The new independent distances $x$ ' may be compared with the $\Lambda$ CDM expanding distances $x$. If the accord is good, this means that the distances by $\Lambda$ CDM expanding model are (as it is obvious) predetermined by the choice of model. Excepting some possible influence of the (arbitrary) parameter $\Lambda$.

The following Table 1A shows the numerical values of the distances $x$ ' obtained multiplying by $(1+\mathrm{z})$ the Static istances $x_{\text {st }}$.

Table 1A. Calculation of the Supernovae artificially expanded distances $x$ ' starting from the Static distances

\begin{tabular}{cccc} 
SN Redshift & CDM model distances & Static model distances & Artif. Expan. distances \\
\hline$z$ & $x$ (Figure 4$)$ & $x_{\text {st }}$ & $x^{\prime}=x_{\text {st }}(1+z)$ \\
\hline$\left.*^{*}\right) 1.91$ & $\approx 4.4 \times 10^{26} \mathrm{~m}$ & $1.44 \times 10^{26} \mathrm{~m}$ & $4.19 \times 10^{26} \mathrm{~m}$ \\
1.76 & 3.88 & 1.37 & 3.78 \\
0.83 & 1.55 & 0.816 & 1.49 \\
0.20 & 0.306 & 0.246 & 0.295 \\
0.10 & 0.159 & 0.148 & 0.163 \\
0.07 & 0.101 & 0.0913 & 0.0977 \\
0.02 & 0.0270 & 0.0267 & 0.0270 \\
0.01 & 0.0135 & 0.0134 & 0.0135 \\
\hline
\end{tabular}

Let's recall that the SNe distances $x$ expressed in logarithmic scale (see Figure 4 and Figure 22, cit.) show an average experimental error around $\pm 2 \%$ and that the fitting curve is not easily readable on the figures, the numerical fitting values of $x$ reported in Table1 may show an uncertainty between $2 \% \div 4 \%$. These values of the SN observations have to be compared with the distances $x$ ' calculated theoretically through the Static model. The discrepancies between $\left(x, x^{\prime}\right)$ are around $3 \%$. We are confident that the proximity between the $\Lambda$ CDM distances $x$ and the independent expanding distances $x$ ' constitute a set of data easily verifiable.

\subsection{Some Early Conclusions}

Our feeling is that the astronomers which claimed the discovery of the "accelerating universe" have actually discovered (thanks to their Supernovae Ia measuring method) that the redshift suffered by photons travelling across the micro-quanta is exponentially increasing along the distance (see Equation 4.3). This fact may appear as an "acceleration" of the motion of galaxies imposed by the expanding model. The novelty may be seen against the decelerating motion required by the orthodox G.R., that describes the gravitational motion within the empty space, i.e. without interaction waves. The Micro-quanta Paradigm appears to penetrate every day new fields of physics. Micro-quanta have shown to be responsible of the Quantum Pushing gravitational force as well as the inertial forces. In particular the strong inertial forces generated by high-energy collisions of heavy charged particles within large accelerators. This fact explains why the energy density of micro-quanta $\left(E_{0} \phi_{\mathrm{o}} / c\right)=2.4 \times 10^{61}\left(\mathrm{~J} / \mathrm{m}^{3}\right)$ is so high. Without this, very high inertial forces couldn't arise immediately in the particle collisions. These ones originate also the high-energy neutrinos adopted in specific experiments. All these phenomena are related to the generation of the Higgs boson (Michelini, 2012).

\section{The Circular Path of the Whole Radiation Emitted in the Universe}

The Static cosmological model assume that at distances $r$ greater than $L_{\mathrm{o}}$, where $\mathrm{G} \exp \left(-r / L_{\mathrm{o}}\right) \approx 0$, the Universe does not sensibly feel the gravitational force. So we may consider static the Universe-at-large. Whereas at distance 
$r<<L_{\mathrm{o}}$ the Universe is quasi-newtonian and undergoes galactic motions, star motions, star gravitational collapses with possible explosions (supernovae). Several observations of astronomic objects show an age consistently higher than 13.7 billions years, a fact that favours the static universe. One of the most intriguing problems of the Big bang expanding model is the mystery of the EM radiation (Visible, Infrared, $\gamma$ rays, $\mathrm{X}$ rays, etc.) that flows from the enormous number of stars $\left(\mathrm{N}_{\mathrm{s}} \approx 10^{21}\right)$, quasars and X-rays sources comprised within the visible universe. Let's recall that photons become "invisible" when their energy is reduced by redshift along travel to such low level that prevents the optical observation. For instance it is sufficient a doubling of the wavelength of sunlight $(z=1)$ to make it invisible. The measurement of the average density within the explored radius $\left(\mathrm{R} \approx 1.7 \times 10^{26}\right)$ has been refined through the recent observations by WMAP 7-years satellite (Jarosik, Bennett et al., 2010) which observed a total equivalent mass density $\rho \approx 9.9 \times 10^{-27}$ shared by atomic matter $(4.49 \%)$, obscure matter $(22.2 \%)$ and obscure energy (72.9\%). The mass ratio (obscure/atomic) equals 4.94. More recently it has been published (Abe, Aghamin et al., 2013) a comparison between the principal parameters measured by "Planck" and "WMAP" satellites. Here are briefly reported the fraction of the atomic (luminous) matter and obscure matter. The fraction coming from the arbitrary parameter $\Lambda$ (extraneous to the static-evolving model) is not reported.

Table 2 . The cosmic mass density measurements by PLANCK and WMAP satellites

Planck (2013 measurement): Atomic matter $4.77 \times 10^{-28}$, Obscure matter $25.5 \times 10^{-27}$ - Mass Ratio $=5.34$
WMAP (9 years measurem.) : Atomic matter $4.6 \times 10^{-28}$, Obscure matter $2.33 \times 10^{-27}$ - Mass Ratio $=5.03$

It appears that the average ratio between Obscure and Atomic matter is consistently around 5. Not interesting is the fraction of Obscure energy which depends on the choice of the arbitrary parameter $\Lambda$.

Let's define the density of the radiation power emitted by all stars $\delta_{\mathrm{P}}=3 \mathrm{~N}_{\mathrm{s}} \mathrm{L}_{\mathrm{o}} / 4 \pi \mathrm{R}^{3}\left(\mathrm{w} / \mathrm{m}^{3}\right)$, where the number of visible stars is $N_{s}=\rho_{o} 4 \pi R^{3} / 3 M_{o}$, while $L_{o}$ is the average-luminosity star and $M_{o}$ its mass. Finally $\rho_{o}=4.6 \times 10^{-28}$ is the average density of luminous mass. Substituting we obtain

$$
\delta_{\mathrm{P}}=\rho_{\mathrm{o}}\left(\mathrm{L}_{\mathrm{o}} / \mathrm{M}_{\mathrm{o}}\right) .
$$

Let's recall the Mass-Luminosity function that relates the luminosity ratio $\left(L / L_{S}\right)$ to the mass ratio $\left(M_{S} / M_{S}\right)$

$$
\mathrm{L} / \mathrm{L}_{\mathrm{s}} \approx\left(\mathrm{M} / \mathrm{M}_{\mathrm{S}}\right)^{4}
$$

where the masses of stars go from 0.43 up to 2 solar masses (Salaris, 2005). Applying this equation to the star with average luminosity $L_{o}$ (and mass $\left.M_{0}\right)$, gives the ratio $\left(L_{o} / M_{0}\right)$ of the average star. Substituting in Equation (5.1) we have the density of power radiation in universe as function of the ratio $\left(\mathrm{M}_{\mathrm{o}} / \mathrm{M}_{\mathrm{S}}\right)$

$$
\delta_{\mathrm{P}}=\rho_{\mathrm{o}}\left(\mathrm{L}_{\mathrm{S}} / \mathrm{M}_{\mathrm{S}}\right)\left(\mathrm{M}_{\mathrm{o}} / \mathrm{M}_{\mathrm{S}}\right)^{3}\left(\mathrm{w} / \mathrm{m}^{3}\right)
$$

where $\left(L_{S} / M_{S}\right)=1.92 \times 10^{-4}$. In literature the average luminosity of stars is often assumed near to the Sun radiation of $3.83 \times 10^{26}$ watts. Rare "blue Giants" are $10^{5}$ times brighter than Sun and might double the average luminosity. On the contrary there are many small stars, whose masses go from $0.1 \mathrm{M}_{\mathrm{S}}$ up to $\mathrm{M}_{\mathrm{S}}$, that are much less luminous than Sun. We leave to skilled astronomers the task of an accurate estimation.

The amount of emitted radiating energy reduces by redshift during flight, while the black body power spectrum remains unchanged (see paragraph 6). What happens to all emitted radiation in the visible universe $\mathrm{N}_{\mathrm{s}} \mathrm{L}_{\mathrm{o}} \approx 10^{48}$ watts disappeared by redshift? What means "disappeared" from the physics standpoint? I think that the correct answer is given by the micro-quanta paradigm which shows that photons are formed by assemblies of great numbers $\mathrm{N}=\mathrm{hv} / \Delta \mathrm{E}_{\mathrm{o}}$ of micro-quanta. Each photon loses energy by removal (see paragraph 4 ) of each bound micro-quanta through collisions with the flux $\phi_{0}$. In this way the energy of all radiation comes back to the "sea" of micro-quanta filling the universe. To this phenomenon corresponds an opposite one that extracts energy from the "sea" of micro-quanta to increase the kinetic energy of oscillating (i.e. accelerating) charged particles (Michelini, 2011). We refer to the phenomenon of the Gravitational power heating the globules of galactic cold gas (Bok's globules) which contract and heat up to the temperature triggering the nuclear fusion reactions that sustain for long time the star radiation. Classical physics has forgotten to explain from where comes the energy that heats the cold gas globules. It is known that Newton's gravitational force between atoms of a cloud works to increase their velocity when moving towards the cloud centre. But the same particles reduce the kinetic energy when moving away from the centre, as any little planet does. Of course the cloud tends to contract because the probability of collision between atoms is greater towards the centre. On the time-average the newtonian force does not furnish 
energy to the gas cloud, because if the radiation comes at the expense of the atom kinetic energy, the temperature of the gas cannot increase. A suggestion has been given (Dragan, 2011) according to which the mass heating is due to the relativistic increase of the atoms falling towards the centre. But the temperature of the Bok's globule is under $100^{\circ} \mathrm{K}$ and the particle velocities are much less the relativistic values. May the cloud heat from loss of the relativistic mass of nuclei? What we know is that the gas cloud heats through the generation of radiation. The heat is probably due to photons emitted during collisions between charged atoms. During the shocking acceleration of the colliding charged nuclei, the emitted photons comes from the "sea" of micro-quanta (Michelini, 2010). This energy may increase the cloud temperature through subsequent adsorption of photons by other atoms of the cloud.

\section{The Power Spectrum of the Redshifted Radiation Coming from All Stars}

There are some similarities between the present cosmology and that known as Stationary model elaborated by Fred Hoyle, H. Bondi. That model accepted the expansion of the universe, but denies the Big bang by assuming the continuous creation of matter (from quantic void) at a rate of about 1 (nucleon $/ \mathrm{m}^{3}$ billion years) to fulfil the Cosmological principle which requires the average density of matter in the universe be substantially constant. A way to create new particles is the production of pairs electron-positron from high energy photon decay. However the rate of formation of new particles seems to be much lower that the amount above recalled. The strongest proof against the Stationary model was the discovery in 1964 of the Cosmic Microwave Radiation (CBR) that was conceptually predicted as the relic of the ambient radiation in the early expanding universe. R.Dicke calculated a radiation temperature of about $46^{\circ} \mathrm{K}$. Penzias \&Wilson measured $\mathrm{T}_{\mathrm{o}}=2.73 \mathrm{~K}$. The importance of the discovery grew when the satellite COBE observed that CBR power flux showed a black body spectrum which was expected due to the ambient temperature of the final interaction between radiation and matter (estimated $\mathrm{T} \approx 3000 \mathrm{~K}$ ) in the Big bang theoretical model.

Let's now calculate in the Static model the background radiation that comes through the redshifted radiation of all stars within the universe. This radiation exists, so its value must be found in whatever cosmological model. The first requirement is to calculate the value of this prediction. The second is to verify that the stellar radiation, which is emitted with a black body spectrum, maintains its form even at the present low temperature $T_{0}$ of the CBR radiation. Let's consider the Planck's black body spectrum of the stellar radiation

$$
\mathrm{I}_{\mathrm{S}}(\mathrm{v})=2 \mathrm{~h} v^{3} / \mathrm{c}^{2}\left[\exp \left(\mathrm{h} v / \mathrm{kT}_{S}\right)-1\right]
$$

where $v$ is the photon frequency and $\mathrm{T}_{\mathrm{S}}$ is the emission temperature of the star. Photons undergo during travel along the distance $x$ the redshift $z_{\mathrm{x}}$, so the arrival frequency is $v_{\mathrm{x}}=v /\left(1+\mathrm{z}_{\mathrm{x}}\right)$. Let's substitute $v$ in Equation (6.1),

$$
\mathrm{I}_{\mathrm{S}}(v)=2 \mathrm{~h} v_{\mathrm{x}}^{3}\left(1+\mathrm{z}_{\mathrm{x}}\right)^{3} / \mathrm{c}^{2}\left\{\exp \left[\mathrm{h} v_{\mathrm{x}}\left(1+\mathrm{z}_{\mathrm{x}}\right) / \mathrm{kT} \mathrm{T}_{S}\right]-1\right\}
$$

Defining $\mathrm{T}_{\mathrm{x}}=\mathrm{T}_{\mathrm{S}} /\left(1+\mathrm{z}_{\mathrm{x}}\right)$ the temperature of the redshifted radiation, one writes

$$
\mathrm{I}_{\mathrm{S}}(v) /\left(1+\mathrm{z}_{\mathrm{x}}\right)^{3}=2 \mathrm{~h} v_{\mathrm{x}}{ }^{3} / \mathrm{c}^{2}\left[\exp \left(\mathrm{h} v_{\mathrm{x}} / \mathrm{kT}_{\mathrm{x}}\right)-1\right]
$$

where

$$
\mathrm{I}(\mathrm{x}, v)=\mathrm{I}_{\mathrm{S}}(v) /\left(1+\mathrm{z}_{\mathrm{x}}\right)^{3}
$$

is the redshifted spectrum at a distance $x$ from the star. Observing that $\exp \left(\mathrm{h} v_{\mathrm{x}} / \mathrm{kT}_{\mathrm{x}}\right)=\exp \left(\mathrm{h} v / \mathrm{kT}_{S}\right)$, one finds by inspection of Equations (6.1) and (6.3) that the whole redshifted spectrum I $(x, v)$ maintains the black body spectrum with a cumulative redshift equal to $\left(1+z_{x}\right)^{3}$. This result shows that the redshift of the whole spectrum differs from the redshift $\left(1+\mathrm{z}_{\mathrm{x}}\right)$ of the single photon. In fact the power flux of the redshifted radiation is obtained integrating on the frequency the black body spectrum of a stellar radiation at a distance $x$ from the star

$$
\int_{o}^{\infty} I(x, v) d v=\mathrm{P}_{i} / 4 \pi x^{2}\left(1+\mathrm{z}_{\mathrm{x}}\right)^{3}\left[\mathrm{w} / \mathrm{m}^{2}\right]
$$

which, recalling Equation (4.3), gives $\left(1+z_{\mathrm{x}}\right)^{3}=\exp \left(3 x / L_{\mathrm{o}}\right)$. Then me may write, recalling Equation (5.2) defining average power density $\delta_{\mathrm{P}}$ of all stars in the universe, the redshifted power density at a distance $x$

$$
\delta_{\mathrm{P}}(x)=\delta_{\mathrm{P}} \exp \left(-3 x / L_{\mathrm{O}}\right)\left[\mathrm{w} / \mathrm{m}^{3}\right] .
$$

This equation introduces explicitly the redshifted power density radiation $\delta_{\mathrm{P}}(x)$. Substituting the known quantities in Equation (5.2) we have

$$
\delta_{\mathrm{P}} \approx 4.6 \times 10^{-28} \cdot 1.92 \times 10^{-4}\left(\mathrm{M}_{\mathrm{o}} / \mathrm{M}_{\mathrm{S}}\right)^{3}=8.83 \times 10^{-32}\left(\mathrm{M}_{\mathrm{o}} / \mathrm{M}_{\mathrm{S}}\right)^{3}\left(\mathrm{w} / \mathrm{m}^{3}\right) .
$$


Assuming this average source of radiation density uniformly distributed let's now sum the contributions on the whole universe of the redshifted radiation density $\delta_{\mathrm{P}} \exp \left[-3 r / L_{0}(x)\right]$ to obtain the All Star Redshifted power flux $p_{A S R}$ calculated in the static-evolving universe, having radius $\mathrm{R}_{m}$ at which the average density of matter $\rho(r)$ equals zero

$$
p_{\mathrm{ASR}}=\int_{o}^{R m}\left(\delta_{\mathrm{p}} 4 \pi r^{2} \mathrm{~d} r\right) \exp \left(-3 r / L_{o}\right) / 4 \pi r^{2}\left(\mathrm{w} / \mathrm{m}^{2}\right)
$$

For clarity, the redshifted radiation density is multiplied by the volume element $\mathrm{dV}=4 \pi r^{2} \mathrm{~d} r$ and divided by the surface $4 \pi r^{2}$ up to which the radiation diffuses. The integration thus supplies

$$
p_{\mathrm{ASR}} \approx \delta_{\mathrm{P}}\left(L_{\mathrm{O}} / 3\right)\left[1-\exp \left(-3 R_{\mathrm{m}} / L_{\mathrm{o}}\right)\right] \text {. }
$$

Observing in Equation (6.6) that when $r=R_{m}$ the redshift takes its maximum effect $\exp \left(-3 R_{\mathrm{m}} / L_{\mathrm{o}}\right)=1 /\left(1+\mathrm{z}_{\mathrm{x}}\right)^{3}$, from Equation (4.3.1) we have $\mathrm{R}_{m} \approx L_{o} \ln \left(1+\mathrm{z}_{\mathrm{x}}\right)=L_{o} \ln \left(\mathrm{T}_{\mathrm{S}} / \mathrm{T}_{\mathrm{o}}\right)=L_{o} \ln (2088)=7.64 L_{o}=10.3 \times 10^{26}$, equivalent to a travel time of 108 billion years. Substituting this value of $\mathrm{R}_{m}$ in Equation (6.7) one shows that the term $\exp \left(-3 R_{\mathrm{m}} / L_{\mathrm{o}}\right)$ is equal to $\exp (-3 \times 7.66)=1 /(2088)^{3}$, which is negligible. Substantially we have that $p_{\mathrm{ASR}} \approx \delta_{\mathrm{P}}\left(L_{\mathrm{O}} / 3\right)$. Substituting $\delta_{\mathrm{P}}$ from Equation (6.5) and $L_{o} \approx 1.35 \times 10^{26}$ from Equation (4.7), one gets

$$
p_{\mathrm{ASR}} \approx 3.97 \times 10^{-6}\left(\mathrm{M}_{\mathrm{O}} / \mathrm{M}_{\mathrm{S}}\right)^{3}\left(\mathrm{w} / \mathrm{m}^{2}\right) .
$$

This value must be compared with the observed CBR power flux $p_{\mathrm{CBR}}=\mathrm{K}_{\mathrm{S}} \mathrm{T}_{\mathrm{O}}^{4}\left(\mathrm{w} / \mathrm{m}^{2}\right)$ where $\mathrm{K}_{\mathrm{S}}$ is the Stefan-Boltzmann constant and $\mathrm{T}_{\mathrm{O}}=2.73 \mathrm{~K}$ is the black body temperature. Substituting one gets the Cosmic Background Radiation power flux

$$
p_{\mathrm{CBR}}=3.15 \times 10^{-6} \mathrm{w} / \mathrm{m}^{2} \text {. }
$$

Comparing now the value of $p_{\mathrm{ASR}}$ power flux with $p_{\mathrm{CBR}}$ it appears that the agreement may be good, depending on the uncertainty of the ratio $\left(\mathrm{M}_{\mathrm{O}} / \mathrm{M}_{\mathrm{S}}\right)^{3}$ between the mass of the average-luminosity star and the mass of Sun, which is expected to be near unity. Let's firstly remark that the assumed value of the mean free path $L_{o}=1.35 \times 10^{26}$ agrees with the CBR measurements directly through Equation (6.6) without elaborated theories on the evolution of an expanding universe. The agreement of $p_{\mathrm{ASR}}$ with $p_{\mathrm{CBR}}$ might be improved by a refined calculation of the mass ratio $\left(\mathrm{M}_{\mathrm{O}} / \mathrm{M}_{\mathrm{S}}\right)^{3}$. Of course it is necessary to follow an independent procedure for selecting the average-luminosity star, a task pertaining to skilled astronomers. To give only an idea of how much accurate would be the common conviction (i.e. Sun luminosity is around the average luminosity among all stars) it is conceivable to calculate the ratio of masses in Equation (6.8) in order to obtain the measured $p_{\mathrm{CBR}}$

$$
\left(\mathrm{M}_{\mathrm{o}} / \mathrm{M}_{\mathrm{S}}\right)^{3}=3.15 / 3.97=0.792
$$

which gives a possible mass $\mathrm{M}_{\mathrm{o}} \approx 0.93 \mathrm{M}_{\mathrm{S}}$ of the average-luminosity star. This mass is close to the solar mass, as expected.

\section{Remarks on the Expanding Model (Big Bang)}

\subsection{Where the CBR is Coming From?}

The Penzias \&Wilson measurements of CBR were firstly used to verify the predictions of the fossil radiation coming from the assumed initial explosion (Big bang). Hence the fossil CBR comprises only the redshifted radiation emitted during the last interaction between photons and electrons at an ambient temperature estimated around $3000 \mathrm{~K}$. Obviously it must be deducted from the measurements the radiation coming from Milky Way and all visible galaxies. However we seriously doubt that is really possible to deduct time by time the redshifted radiation coming from all galaxies comprised within the radius presently explored by WMAP satellite. The estimate through Eqauation (6.6) of the amount of redshifted radiation coming from the stars comprised within the radius $\mathrm{R}_{\mathrm{v}} \approx 1.7 \times 10^{26}$ is given by

$$
p^{\prime}{ }_{\mathrm{ASR}}=\delta_{\mathrm{P}} \int_{o}^{R_{v}} \exp \left(-3 x / L_{o}\right) \mathrm{d} r \approx \delta_{\mathrm{P}}\left(L_{\mathrm{O}} / 3\right)\left[1-\exp \left(-3 \mathrm{R}_{\mathrm{v}} / L_{\mathrm{o}}\right)\right]
$$

which differs quantitatively from Equation (6.7) only in the term $\left[1-\exp \left(-3 \mathrm{R}_{\mathrm{v}} / L_{\mathrm{o}}\right)\right]$. Calculating the exponential, one gets the power flux to be deduced from the CBR measurement

$$
p_{\text {ASR }}^{\prime}=\mathrm{p}_{\mathrm{ASR}} 0.977 \text {. }
$$

This shows that the redshifted radiation from all visible stars $\mathrm{p}_{\mathrm{ASR}}$ represents $\approx 97.7 \%$ of the measured $\mathrm{p}_{\mathrm{CBR}}$. In other words a profound correction of the measured CBR power flux by statistical deduction of all spurious 
contributions would make negligible the fossil radiation from the Big bang, which never happened. This proves that the CBR measurement sustains the Static-evolving universe.

\subsection{Other Problems of the $\Lambda C D M$ Model}

Beyond the above fundamental proof that discards the Big bang model, this one presents several problems of different nature. Neglecting the problem of the cosmic Inflation that appears hardly based on the known laws of physics, the second question regards the so called Dark matter which, according to the proponents of the $\Lambda \mathrm{CDM}$ model, is not made of baryons but of particles not yet identified which do not emit radiation. There is an excessive use of strange hypotheses to hide the reality of dense obscure masses that is well known to the astronomers. We remain on the Vera Rubin's idea that Dark matter is made of ordinary matter whose physical conditions don't consent emissions so luminous to be observed at large distances. Neutron stars and White dwarf show a life (cooling time) much higher than 14 billions years. The dark matter resulting from the WMAP measurements equals 5 times the total luminous mass in the universe. We assume this value for the obscure mass present in the Milky Way, that may be likely constituted of dense old stars (such as Neutron stars, White and Brown dwarfs, pulsars, etc) that show very low luminosity due to their small emitting surface. The radius $R_{\mathrm{D}}$ may go from $4 \times 10^{7}$ for W\&B dwarfs, down to $10^{5} \div 2 \times 10^{4}$ for dense Neutron stars, while their masses may largely exceed the mass of Sun due to the gravitational accretion from the surrounding galactic gas (during a time of the order of several tens of billion years) or from the stellar companion. In particular several Supermasses due to accretion have been discovered in the galactic nuclei. For instance in the nucleus of the Milky Way a supermass of about $3 \times 10^{6}$ Sun masses has been discovered in Sagittarius A*(Schoedel et al., 2002). The gravitational redshift of the obscure masses

$$
z=\left(2 \mathrm{G} / \mathrm{c}^{2}\right)(\mathrm{M} / \mathrm{R})_{\mathrm{D}}
$$

may concur in reducing the luminosity through the high values (up to $10^{3}$ for supermasses) of dense obscure masses having ratios $(\mathrm{M} / \mathrm{R})_{\mathrm{D}} \approx(0.7 \div 5) \times 10^{27}$. The total mass of the obscure dense stars present in the Milky Way has been estimated in the past by Jan Oort (1932) to be $6 \div 7$ times the known galactic mass by measuring the high velocity of stars orbiting the external galaxy. Observing the rotational speeds of galaxies, Vera Rubin produced in the ' 70 a clear observational evidence that the vast bulk of the mass in the universe is obscure (invisible). According to the observations of the WMAP and Planck satellites (see par. 4) the obscure mass in the universe equals about 5 times the luminous atomic mass. Assuming this factor to be valid also for our galaxy, the whole obscure mass likely amounts to about 5 times the mass of the $10^{11}$ luminous stars, provided the age of universe is long enough (about 100 billion years) that the Neutron stars originated by sudden collapse of supernovae may go up to $10^{10}$ at a rate of about 0.1 supernova observed yearly (CBAT, 2013) in our galaxy. The exiting luminous stars evolve from the Main sequence towards the obscure W\&B dwarfs at a rate likely of $5 \div 8$ stars per year. A little fraction of the "dark" stars reveal their existence by accreting mass from the surrounding galactic gas, which is accelerated near the velocity of light giving rise to large emissions of luminous radiation and X-rays. The great luminosity of these quasi-stellar objects (Quasars) have been often equivocated for emissions of galaxies (Lopez-Corredoira, 2011) since their high gravitational redshifts have been interpreted as cosmological redshifts.

\subsection{The Theoretical Problem Created by the ACDM Cosmological Model}

What creates the greatest theoretical trouble with the $\Lambda \mathrm{CDM}$ cosmological Model, which inherited the difficult task of solving the problems accumulated on the last century of observations, is the high level of arbitrariness presented by this Model which has at disposal some parameters to be adjusted to the aim of building new models able to fit new observations. The inadequacy of the original G.R. to face cosmological phenomena (solved by Quantum Pushing Gravity) is now focussed towards a new parameter, the Obscure energy, whose physical significance is dubious. The chance of discovering the original inadequacy of G.R. is practically null.

Let's recall the objective basic assumptions of the $\Lambda$ CDM model:

1) The Cosmic Microwave Background with its peculiar spectrum is the relic of the Big bang,

2) The Hubble's redshift of galaxies comes from an initial cosmic explosive expansion which left the calculated abundance of hydrogen, helium and lithium in the universe

3) The obscure matter is made of unknown particles without EM emissions, but with gravitational properties,

4) The observation of the accelerating universe is considered an advancement on the present Cosmology.

Some general considerations through comparison between $\Lambda$ CDM model and Static model.

1) The measurements of the CMB are fundamental in proofing expanding models, which consider negligible the spurious contribution from the stars. In the Static-evolving model the CMB is demonstrated to come from the 
redshifted radiation of all stars of universe.

2) In Static model no hypothetical initial cosmic expansion is needed to explain the redshift of distant galaxies which is due to the interaction of photons during travel across the flux of micro-quanta. The calculations about the production of the lightest elements are shared from the thermonuclear reactions feeding the stars. The Expanding model does not predict the high metallicity of primordial Quasars. In the Static-evolving model the nucleo-syntesis happens entirely within the cores of stars. Starting from the hydrogen of large clouds diffuse in the universe (which become proto-stars through the gravitational collapses of Bok's globules), the above reactions are obviously complemented with the synthesis of the heavy metals within the cores of massive stars.

3) The existence of a large amount of obscure baryonic matter trapped in obscure dense masses is not a novelty from several decades. The news is that WMAP and PLANCK satellites found that Obscure matter is about 5 times the luminous matter, as proposed by Vera Rubin in the ' 70 .

4) The observation of an accelerated expansion in a part of the universe, compared to the deceleration of the remaining part, show a change in the usual predictions of G.R. This change is considered dependent on the arbitrary term $\Lambda$. The fact that Einstein did not found a better theory to correctly modify the Newton's force at high cosmological distances, does not authorise to assign arbitrary values to the constant $\Lambda$.

The $\Lambda$ CDM accelerating effect may be better understood through the words of J. Hartnett: "In order for the observations to fit the standard cosmology it has been necessary to add dark energy with a non-zero value for the cosmological constant $\Lambda$ and also a significant amount of dark matter." (Hartnett, 2011). These words confirm that to obtain advancements in cosmology it is necessary to reject the possibility of adjusting empirical parameters. Cosmological Models without free parameters are, when possible, preferable.

Trying to resume the situation about the $\Lambda \mathrm{CDM}$ model we quote a neutral observer (Wikipedia): "There is currently active research into many aspects of the $\Lambda C D M$ model, both to refine the parameters and possibly detect deviations. In addition $\Lambda C D M$ has no explicit physical theory for the origin of physical nature of Dark matter or Dark energy".

\section{An Application of the New Cosmological Static Model Discussed in the Introduction}

An application of the Static model arising from the Quantum Pushing gravity may be done introducing in the dynamical equilibrium of the masses of the universe the changes discussed in the Introduction, i.e. cancelling the arbitrary cosmological constant $\Lambda$ and substituting the uniform $\mathrm{G}$ with the variable $\mathrm{G}(r)=\mathrm{G} \exp \left(-\Sigma_{\mathrm{o}} r\right)$ across the material universe.

Firstly it is necessary to give a brief description of the universe as we saw it. A century of astronomical observations gave us the knowledge of an amount of about $10^{10}$ galaxies populating the visible universe, often assembled in clusters of hundreds of galaxies. In the visible universe, galaxies appear distributed randomly, but observations confirm that the average density of mass (within a sphere about $10^{24}$ metre in diameter) appears about uniform across the universe. We don't know where is the centre of mass of the universe, although recent claims on the asymmetric distribution of the far galaxies may give indications about this. Anyway we know that each galaxy (or cluster of galaxies) at a distance $r$ from the centre, is orbiting around it with a tangential velocity $v$ and a radial acceleration $v^{2} / r$. In the new "static" Cosmology the masses orbiting at distance $r$ are bound by the Quantum pushing gravitation force (Equation 2.7) to the mass of all galaxies orbiting at distances less than $r$. The equilibrium takes place between the gravitational force and the inertial/centrifugal forces, which now have physical nature arising not from empty space, but from the motion of masses across the flux of micro-quanta (Michelini, 2012). In this frame we assume the average mass density $\rho_{o}=2.76 \times 10^{-27}$ obtained by VMAP 9-years measurement (see Table 2 ) by multiplying the total density $9.9 \times 10^{-27}$ by the sum of Atomic luminous matter (4.63\%) and Dark matter $(23.3 \%)$

$$
(4 \pi / 3) \mathrm{G} \exp \left(-\Sigma_{o} r\right) \rho_{\mathrm{o}} r=v^{2} / r .
$$

Some galaxies orbit at a distance $R$ with the maximum velocity $v_{M}^{2}$, according to the equation

$$
(4 \pi / 3) \mathrm{G} \exp \left(-\Sigma_{o} R\right) R^{2} \rho_{\mathrm{o}}=v_{M}^{2} .
$$

Being $R_{*}=2 / \Sigma_{o}$ the distance where the function $\exp \left(-\Sigma_{o} R\right) R^{2}$ takes the maximum, one has to calculate $v_{M}{ }^{2}$ to verify that $v_{M}<\mathrm{c}$. Substituting in Equation (8.2) $R_{*}$ and the known quantities, we have

$$
v_{M}^{2}=(4 \pi / 3) \rho_{\mathrm{o}} \mathrm{G} \exp (-2) 4 L_{\mathrm{o}}^{2}=0.625 \times 10^{16} .
$$

The ratio $v_{M}{ }^{2} / \mathrm{c}^{2}$ corresponds to $\beta=0.964$. This confirms that Equation (8.1) has a different physical nature from the old Einstein static model, whose equation is not useful to give the radius of the material universe. Photons travel at 
light velocity and undergo the gravitational force, but the high redshift they suffer travelling across the flux of micro-quanta reduces all orbiting photons to degraded CBR waves before they can complete the first orbit.

In Paragraph 7 it has been shown that the stellar radiation is degraded up to CBR during the travel on a distance which resulted to be $X=L_{o} \ln \left(1+\mathrm{z}_{x}\right)$, where the redshift $\mathrm{z}_{x}=\left(\mathrm{T}_{S} / \mathrm{T}_{o}\right)=2087$ is the ratio between the emision temperature of star $\mathrm{T}_{S}$ and the equivalent temperature $\mathrm{T}_{o}$ of the CBR. The distance $X$ is near the radius of the material universe $\mathrm{R}_{m}$, which has been calculated from eq(9.2) through a convenient assumption on the radius $\mathrm{R}_{u}$ of the Grand Cloud of micro-quanta that envelope the material universe. Finally the radius resulted $\mathrm{R}_{m} \approx 8.57 L_{0}$.

Equation (8.1) gives for any galaxy at a distance $r$ the rotation velocity $\omega=v / r$

$$
(4 \pi / 3) \mathrm{G} \exp \left(-\Sigma_{o} r\right) \rho_{\mathrm{o}}=\omega^{2}(r) \text {. }
$$

It may appear strange to name "Static universe" this New model which shows a non zero rotation of all galaxies orbiting on different planes. The correct name should be "Non-expanding universe". In practice the task of observing a differential rotation between galaxies appears possible. Detecting the difference of rotations of distant galaxies placed on the same line of sight is however quite difficult. The highest rotation velocity $\omega_{\mathrm{M}} \approx 1.6 \times 10^{-18}$ requires 120 Gys to perform a complete rotation. The distant galaxies at periphery of the material universe show a rotation velocity equal to $\omega=\omega_{\mathrm{M}} \exp \left(-R_{m} / L_{o}\right) \approx \omega_{\mathrm{M}} / 2000$ which requires $2.4 \times 10^{5}$ Gys to complete 1 rotation. These galaxies, practically static, would permit the observation of differential rotations of nearest galaxies. But they are invisible because their radiation suffered a redshift of the order of $\mathrm{z}_{\mathrm{M}} \approx 2000^{3}$ and arrives as CBR waves. A better situation occurs when we observe two galaxies at the same distance, but with opposite rotation, a situation that the astronomers called the merger of Galaxies. In this case the time needed to observe a complete merger of two galaxies with diameter $\approx 10^{19}$ is of the order of $3.6 \times 10^{3}$ years. To observe only a merger of $1 \%$ the time needed is about 36 years. Someone will surely imagine some better way to observe the slow galaxy rotations characterising the Static universe. A possible way may be to calculate the orbital velocity of a galaxy through Equation (8.1)

$$
\mathrm{v}^{2}=(4 \pi / 3) \mathrm{G} \exp \left(-\Sigma_{\mathrm{o}} r\right) \rho_{\mathrm{o}} r^{2}
$$

which, recalling Equation (4.3) and substituting the distance in Static universe $r=L_{\mathrm{o}} \ln (1+\mathrm{z})$, gives

$$
v^{2}=(4 \pi / 3) \mathrm{G} \rho_{\mathrm{o}} L_{o}{ }^{2} \ln ^{2}(1+\mathrm{z}) /(1+\mathrm{z})
$$

This equation furnishes the orbital velocity of galaxies through a simple measurement of the redshift.

\section{Some Hypotheses on the Effects of the Finite Size of Static Universe}

The reducing gravitational constant in the new Static model determines an universe extension notably larger than in the G.R. model. Besides in Equations (8.3-8.4) it may be necessary to assume an average density $\rho_{o}(r)$ which goes to zero at the periphery $R_{m}$ of the material universe. This fact requires that also the flux $\phi(r)$ depends in some way from the distribution of $\rho_{o}(r)$ because the M-Q flux tends to crowd near the dense matter. The variation of $\phi(r)$ influences the macroscopic cross section of micro-quanta $\Sigma_{0}(r)=\sigma_{0} \phi(r) / \mathrm{c}$ which tends to vanish at the periphery of the Grand Cloud of micro-quanta (radius $R_{u}$ ). This depends on the hypotheses we shall make about the functions $\rho_{o}(r)$ and $\phi(r)$.

For instance, let's the flux assumes the form $\phi(r)=\phi_{\mathrm{o}} \cos \left(\pi r / 2 R_{u}\right)$. The average value $\Sigma_{\mathrm{m}}$ of the cross section $\Sigma(r)$ along the radius $R_{m}$ is defined through the optical distance

$$
\xi_{\mathrm{m}}=\underline{\Sigma}_{\mathrm{m}} R_{m}=\int_{0}^{R_{m}} \sigma_{0} \phi_{0}(r) \mathrm{d} r / \mathrm{c}=\Sigma_{\mathrm{o}} \int_{0}^{R_{m}} \cos \left(\pi r / 2 R_{u}\right) \mathrm{d} r=\Sigma_{\mathrm{o}}\left(2 R_{u} / \pi\right) \sin \left(\pi R_{m} / 2 R_{u}\right)
$$

Putting the angle $\alpha=\left(\pi R_{m} / 2 R_{u}\right)$ the preceding equation becomes

$$
\xi_{\mathrm{m}}=\Sigma_{\mathrm{o}} R_{\mathrm{m}}(\sin \alpha / \alpha) .
$$

By virtue of Equation (4.3a) we have $\xi_{\mathrm{m}}=\ln \left[1+z_{\mathrm{m}}\right]=7.64=\Sigma_{\mathrm{o}} \mathrm{R}_{\mathrm{m}}(\sin \alpha / \alpha)$ which gives

$$
R_{m}=7.64 L_{\mathrm{o}}\left(\pi R_{m} / 2 R_{u}\right) / \sin \left(\pi R_{m} / 2 R_{u}\right)
$$

This equation may be solved assuming that the radius $R_{u}$ of the Grand Cloud exceeds the radius $R_{m}$ of about 6-8 mean free path $L_{o}$, that is $R_{u} \approx(7.64+7) L_{o} \approx 14.6 L_{o}$ which gives the ratio $R_{m} / R_{u} \approx 0.523$ to be put into eq $(9.2)$. Finally one has the improved radius of the material universe

$$
R_{m} \approx 8.57 L_{0} \text {. }
$$

This procedure can be generalised to the static distance substituting $x_{\mathrm{st}}=L_{o} \ln [1+\mathrm{z}(x)]$ to the radius $R_{m}$ in Equation 
(9.2) thus obtaining the improved distance

$$
x^{\prime}{ }_{s t}=L_{o} \ln (1+\mathrm{z})\left[\left(\pi x_{\mathrm{st}} / 2 \mathrm{R}_{\mathrm{u}}\right) / \sin \left(\pi x_{\mathrm{st}} / 2 \mathrm{R}_{\mathrm{u}}\right)\right]=L_{o} \ln (1+\mathrm{z}) \alpha(x) / \sin \alpha(x) .
$$

The calculation of $x^{\prime}{ }_{s t}$ may be simplified by substituting within brackets $x_{\mathrm{st}} \approx L_{\mathrm{o}} \ln [1+z(x)]$ so obtaining the ratio $\alpha(x) / \sin \alpha(x)$ where $\alpha(x)=(\pi / 2) \ln (1+z)\left(L_{o} / R_{u}\right)$. Being $L_{o} / R_{m}=1 / 8.57$ and recalling the assumption $R_{m} / R_{u} \approx 0.523$ one gets $\left(L_{\delta} / R_{u}\right)=0.523 / 8.57=0.061$ which may be substituted in the ratio

$$
\alpha(\mathrm{x}) / \sin \alpha(\mathrm{x})=0.061(\pi / 2) \ln (1+\mathrm{z}) / \sin [0.061(\pi / 2) \ln (1+\mathrm{z})]
$$

Considering that $\alpha(\mathrm{x})=0.061(\pi / 2) \ln (1+\mathrm{z})<<1$, developing in power series one has $\sin \alpha(\mathrm{x}) / \alpha(\mathrm{x}) \cong 1-\alpha^{2}(\mathrm{x}) / 6$.

Substituting in Equation (9.3) we obtain

$$
x_{s t}^{\prime} \approx L_{o} \ln (1+z) /\left[1-0.0171 \ln ^{2}(1+z)\right] .
$$

In Table 3 is reported the improved value $x^{\prime}{ }_{s t}$. The refinement is so little to justify the approximations adopted for reaching Equation (9.4).

Table 3. Redshifts and distances $x_{s t}$ taken from Table 1 put in comparison with the distances calculated in the Static model with a finite radius $R_{\mathrm{m}}$ of the material universe

\begin{tabular}{cccc}
\hline Redshift & Optical thickness & Static distances & Static distances $x_{\text {st }}{ }^{\prime}$ \\
\hline$z$ & $\xi(x)=\ln (1+z)$ & $x_{s t}=L_{o} \ln (1+z)$ & $\left(\right.$ finite radius $\left.R_{m}\right)$ \\
\hline$\left.{ }^{*}\right) 1.91$ & 1.067 & $1.44 \times 10^{26}$ & $1.468 \times 10^{26}$ \\
1.76 & 1.016 & 1.37 & 1.394 \\
0.83 & 0.604 & 0.816 & 0.820 \\
0.20 & 0.182 & 0.246 & 0.246 \\
0.10 & 0.0953 & 0.128 & 0.128 \\
0.07 & 0.0675 & 0.0910 & 0.0910 \\
0.02 & 0.0198 & 0.0268 & 0.0268 \\
\hline
\end{tabular}

\section{References}

Abe, P., Aghamin, N., Armitage-Caplan, C., Arnaud, M., Ashdown, M., Atrio-Barandela F., \&Zonca A. (2013). Planck Collaboration. Retrieved from arxiv:1303.5076v1-2013-astro-ph.CO, 2013.

Carroll, S. (2001). The Cosmological Constant. Living Rev. Relat., 3, 1.

CBAT. (1999-2010). List of Possible Supernovae. http://www.cbat.eps.harward,esu/CBAT_PSN.html

Clocchiatti, A., Schmidt, B. P., Filippenko, A. V., Challis, P., Coil, A. L., \& Covarrubias, R. (2006). Hubble Space Telescope and Ground-based Observations of Type Ia Supernovae at Redshift 0.5: Cosmological implications. The Astrophysical Journal, 642(1).

Dragan, V. (2011). A paradoxical Deviation from Lavoisier's Postulate using the Classic Newtonian Gravity. Applied Physics Research, 3(2), 203. http://dx.doi.org/10.5539/apr.v3n2p203

Hubble, E. P. (1929). A Relation berween Distance and Radial Velocity among Extra-Galactic Nebulae. Proc. Natl. Acad. Sci. USA, 15, 168-173. http://dx.doi.org/10.1073/pnas.15.3.168

Jarosik, N., Bennett, C. et al. (2010). (WMAP collaboration) Seven-Year Wilkinson Microwave Anisotropy Probe (WMAP) Observations. Retrieved from http://lambda.gsfc.nasa.gov/product/map/dr4/pub_papers

Jones, Rodney, \& Riess. (2013). The Discovery of the most Distant known Type Ia Supernova at Redshift 1.914. Retrieved from arxiv:1304.0768v2 [astro-ph.CO], April, 2013.

Lineweaver, C. H., \& Davis, T. M. (2005). Misconceptions about the Big bang. Sci. Am. J., Febr. 21.

Lopez-Corredoira, M. (2011). Pending Problems on QSOs. Int. Jr. Astron.\&Astroph., 1, 73-82. 
Michelini, M. (2012). Beyond the Special and General Relativity Theories. The Micro-quanta Paradigm. Applied Physics Research, 4(2), 210. http://dx.doi.org/10.5539/apr.v4n2p210

Michelini, M. (2010a). Major gravitational Phenomena Explained by the Micro-quanta paradigm. Progr. In Physics, 1, Jan. 2010

Michelini, M. (2010b). Physical Phenomena and Theoretical Problems explained by the Micro-quanta Paradigm. Applied Physics Research, 2(2).

Michelini, M. (2011). The Gravitational Power Originating the Earthquakes and the Dansgaard-Hoeschger Events. Applied Physics Research, 3(1), 76. http://dx.doi.org/10.5539/apr.v3n1p76

NASA/ESA. (2013). Hubble Breaks Records for Furthest Supernova. Hubble Sp. Tel. Retrieved April 4, 2013 from www.spacetelescope.org/news/heic1306/

Perlmutter S. et al. (1999). The Astroph. Journal Nobel lecture for Physics.

Riess, A. G., Strolger, L.-G., Tonry, J., Casertano, S., Ferguson, H. C., Mobasher, B., ... Tsvetanov, Z. (2004). Type Ia Supenova Discoveries at $\mathrm{z}>1$ from the Hubble Space Telescope: Evidence for Past Deceleration and Constraints on Dark Energy Evolution. The Astrophysical Journal, 607, 665-687.

Salaris, M., \& Santi, C. (2005). Evolution of stars and stellar populations (pp. 138-140). John Wiley \& Sons.

Schödel, R., Ott, T., Genzel, R., Hofmann, R., Lehnert, M., Eckart, A., ... Menten, K. M. (2002). A star in a 15.2-year orbit around the supermassive black hole at the centre of the Milky Way. Nature, 419, 694-696.

Schwartz, D., \& Weinhorst, B. (2007). Anisotropy of the Hubble diagram :comparing hemispheres. Retrieved from arXiv:0706.0165 [astro-ph], 2007.

Wang, L. J. (2011). Saturated dispersive extintion theory of the redshift. Physics Essays, 24(4), Dec. 2011. http://dx.doi.org/10.4006/1.3651528

Zaninetti, L. (2010). New formulae for the Hubble constant in a Euclidean Static Universe. Retrieved from arxiv:1005.0263v1[astro - ph CO], 3 May, 2010.

\section{Copyrights}

Copyright for this article is retained by the author(s), with first publication rights granted to the journal.

This is an open-access article distributed under the terms and conditions of the Creative Commons Attribution license (http://creativecommons.org/licenses/by/3.0/). 